\title{
Tattoos, scars, body adornment and dishevelment in an acute psychiatric population
}

\author{
Kelwyn Williams
}

\begin{abstract}
Ninety-six successive acute psychiatric admissions were assessed with regard to dress, level of hyglene, the presence of taltoos, bodily plercing/decoration and surface marks of self-harm. These features were also examined in relation to ICD-10 category diagnosis. One-third were described as 'dishevelled', 21\% 'unclean', 7\% had multiple plerced ears and 3\% other plerced body parts. Sixteen per cent had tattoos, rising to $34 \%$ in the F10-19 (substance misuse) category, and $15 \%$ had scars of self-harm. People with schizophrenia and related disorders were more likely to present in an unciean state. Those with personality disorders were no more likely to bear the scars of self-harm, fattoos or body plercing.
\end{abstract}

In classical psychiatric teaching certain modes of dress or states of hyglene are associated with types of psychiatric diagnosis. Self-neglect and an unkempt look may suggest alcoholism, depression or schizophrenia. The manic patient may wear bright colours, adopt incongruous styles of dress or appear poorly groomed. An oddity of dress may provide a clue to diagnosis (Gelder et al, 1996). Scars of self-harm, tattooing and body piercing are often associated with diagnosis of personality disorders or substance misuse. More specific correlations have been proposed: Howard \& Valori (1989) suggested a link between patients who wear sunglasses and psycho-neurosis.

\section{The study}

For every acute adult (age range 18 to 65) admission to Wotton Lawn Hospital, Gloucester, over three months from October 1995, a questionnaire was filled in by the admitting doctor. Questionnaires included demographic details, and the physician's impression of the patient's state of hygiene (fair or unclean), state of dress (fair or dishevelled), number and site of ear or other body piercing, presence of tattoos and the nature and quality of any self-inflicted scars. Any other abnormality of appearance could be recorded. At discharge consultant diagnosis by ICD-10 category was added.

\section{Findings}

Over the study period, there were 96 admissions in the appropriate age group. Questionnaires were returned on all subjects. Breakdown by main ICD-10 diagnostic category is shown in Table 1.

The mean age was 37.2 years (s.d. 11.4). Flftyeight out of the ninety-six patients were male. The mean male age was 36.3 (s.d. 11.6, range 19-62) and the mean female age 37.8 (s.d. 11.7. range 18-64).

Table 1. Diagnostic categories of admission

\begin{tabular}{lr}
\hline & $n$ \\
\hline F00-09 (Organic mental disorders) & 1 \\
F10-19 (Disorders due to psychoactive substance misuse) & 29 \\
F20-29 (Schizophrenia, schizotypal and delusional disorders) & 18 \\
F30-39 (Mood disorders) & 22 \\
F40-48 (Neurotic, stress related and somatoform disorders) & 14 \\
F50-59 (Behavioural syndromes associated with psychological disturbances and physical features) & 0 \\
F60-69 (Disorders of adult personality and behaviour) & 9 \\
F70-79 (Mental retardation) & 1 \\
Others & 2 \\
\hline
\end{tabular}




\section{Hygiene}

Twenty (21\%) of the patients admitted were rated 'unclean' by the assessing doctor $(22 \%$ of the males and $18 \%$ of females). Twenty-eight per cent of patients with the F10-19 diagnosis group were considered unclean as were a similar percentage of the F20-29 group.

\section{Dishevelment}

Thirty-two $(33 \%)$ of the patients appeared dishevelled, $36 \%$ of the males and $29 \%$ of females. Dishevelment was spread evenly across the diagnostic groups with a slight excess (5 out of 9) in those with personality disorders.

\section{Multiple pierced ears}

Seven $(7 \%)$ of the patients had multiple pierced ears, 3 males and 4 females. This variable was distributed evenly across the diagnostic categories.

\section{Other body piercing}

Three (3\%) of the patients had other areas of their body pierced. In two cases the pierced area was a nostril (both female). In the third individual, a male, there were 18 pierced areas, including nipples and genitals. His diagnosis was of depressive disorder.

\section{Tattoos}

Fifteen patients (16\%) had tattoos. These varied from self-inflicted dots, crosses and 'Love/Hate' knuckle motifs, to elaborate pictorial and heraldic representations. One patient had most of his upper torso covered in floral patterns and the names of friends and relatives in calligraphic flourishes. Twenty-two per cent of male patients bore tattoos compared with $5 \%$ of females. They were present in $34 \%$ of patients in the substance misuse group but only in one out of the nine patients in the personality disorder category.

\section{Beards}

Ten out of 96 admissions ( $10 \%$ ), all male, sported beards, broadly spread over all diagnostic categories.

\section{Scars}

Fourteen of the admissions bore marks of selfharm (15\%). While most were superficial marks to the wrists, in four cases the scars were extensive and deep, including in two cases, cigarette burns to the abdomen and forearms. Six of the 14 scarred patients were male $(10 \%$ of total males). The eight scarred females comprised $21 \%$ of the total female sample. Mean age of scarrers was $\mathbf{3 0}$ years compared with $\mathbf{3 7}$ for all admission and breakdown by diagnostic category showed that scarring was represented evenly across all groups. Two out of the nine cases were in the personality disorder category.

\section{Bare feet}

Seven out of the 96 were barefooted, three of the seven being in the F10-19 (substance misuse) grouping.

\section{Other}

Two patients wore hats and none tinted sunglasses.

\section{Comment}

Owing to limitations of methodology, the results of the survey must be interpreted in a limited context. It was an uncontrolled, naturalistic study of consecutive acute psychiatric admissions and there was no reference to community control samples. Certain of the assessments, such as the state of hygiene, were subjective, with an all or nothing response. Other shortcomings include the limitations in the search that the admitting doctor may have made for covert body piercing or tattoos. Cultural factors, which may influence the willingness to disrobe. may have confounded the assessment. It is also possible (although ideally not desirable) that the ICD diagnosis made by the clinical team had been influenced by nuances of appearance.

None the less some findings are worthy of discussion. The study confirmed a clinical impression that a poor state of hygiene and dishevelled appearance are common to many patients presenting with acute mental illness. Tattooing and scars of self-harm seemed common. Breakdown by diagnostic category failed to show an expected association between personality disorder and body piercing, scars of selfharm and tattooing despite antisocial personality disorder, substance misuse and the borderline personality disorders having been reported to be associated with tattoos (Raspa \& Cusack, 1990). More specifically, reports have linked tattooed female patients to borderline personality disorder and childhood sexual abuse (Inch \& Huws. 1993). Self-mutilation was found to be associated with a history of substance misuse and axis II diagnoses (Langbehn \& Pfohl, 1993). Ali (1990) proposed that the borderline personality disorder was associated with multiple ear-lobe piercing.

Much of the previous literature was of limited validity, being based largely on anecdotal case reports rather than survey material. 
The findings from this survey suggest that commonly held clinical beliefs in certain associations may have poor foundation, and there is non-specificity in the association between nuances of appearance and clinical diagnosis.

More comprehensive cross-sectional hospital and comparative population studies may be useful.

\section{Acknowledgements}

The author thanks psychiatric trainees in Severn NHS Trust for participating in the collection of the data, Kathy Humphries in the Health Records Department for extracting the ICD-10 diagnoses and Rob Macpherson for advice with the project and help in preparing the manuscript.

\section{References}

Au. A. H. (1990) Borderline personality and multiple earrings a possible correlation? (letter). American Journal of Psychiatry, 147, 1251.

GelDER, M.. GATH, D.. MAYou, R., et al (1996) Oxford Textbook of Psychiatry, p. 33. Oxford: Oxford University Press.

HOWARD, R. J. \& VALORI, R. M. (1989) Hospital patients who wear tinted spectacles - physical sign of psychoneurosis: a controlled study. Joumal of the Royal Society of Medicine, 82, 606-608.

INCH, H. \& Huws, R. (1993) Tattooed female psychiatric patients. British Joumal of Psychiatry, 163, 127-128.

LANGBEHN, D. R. \& PFOHL, N. B. (1993) Clinical correlates of self mutilation among psychiatric inpatients. Annals of Clinical Psychiatry, 5, 45-51.

RASPA, R. F. \& CUSACK, J. (1990) Psychiatric implications of tattoos. American Family Physician, 41, 1481-1486.

Kelwyn Williams, Specialist Registrar in Community Psychiatry. Wotton Lawn Hospital, Horton Road, Gloucester GL1 3PX 